\section{Clinical utilization of shear wave elastography in the musculoskeletal system}

\author{
Leah C. Davis ${ }^{1,2}$, Timothy G. Baumer ${ }^{3}$, Michael J. Bey ${ }^{3}$, Marnix van Holsbeeck ${ }^{2}$ \\ 'Department of Radiology and Radiological Sciences, Medical University of South Carolina, \\ Charleston, SC; ${ }^{2}$ Department of Radiology, Henry Ford Health Systems, Detroit, MI; \\ ${ }^{3}$ Department of Orthopaedic Surgery, Henry Ford Health Systems, Bone and Joint Center, \\ Detroit, MI, USA
}

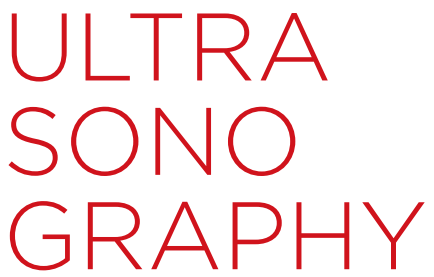

REVIEW ARTICLE

https://doi.org/10.14366/usg.18039 pISSN: 2288-5919 • elSSN: 2288-5943 Ultrasonography 2019;38:2-12

Received: July 25, 2018

Revised: August 22, 2018

Accepted: August 23, 2018

Correspondence to: Marnix van Holsbeeck, MD, Department of Radiology, Henry Ford Health Systems, 2799 West Grand Blvd, Detroit, MI 48202, USA

Tel. +1-313-623-7329

Fax. +1-313-916-9475

E-mail: marnix@rad.hfh.edu

\title{
Introduction
}

Since the first sonographic images of the musculoskeletal system were obtained in 1958 [1], ultrasonography has become an important imaging modality in musculoskeletal radiology. Its inherent strengths of high spatial resolution, dynamic imaging capabilities, ability to assess vascular flow without intravenous contrast, and relatively low cost have established ultrasound as an irreplaceable modality in musculoskeletal imaging [2]. With the advent of high-frequency transducers, wide/ panoramic fields of view, and improved sensitivity of Doppler imaging, ultrasound images are able to provide more detailed anatomic information about structures in the musculoskeletal system than ever before. Despite these technological advances, gray-scale and color Doppler images are unable to provide any biomechanical information about tendon quality [3]. Ultrasound elastography is an emerging technology that provides information about the inherent elasticity of tissues and has the potential to detect tissue changes induced by trauma, degeneration, healing, or tumors. Two types of elastography are currently available for clinical use in ultrasonography: strain or pressure elastography and shear wave elastography.

Strain elastography applies longitudinal or compressive waves to evaluate differences in tissue strain, defined as deformation of the size or shape of tissues, as a result of the stress applied. This provides qualitative data in the form of color maps and semiquantitative data by calculating the
This is an Open Access article distributed under the terms of the Creative Commons Attribution NonCommercial License (http://creativecommons.org/ licenses/by-nc/3.0/) which permits unrestricted noncommercial use, distribution, and reproduction in any medium, provided the original work is properly cited

Copyright (C) 2019 Korean Society of Ultrasound in Medicine (KSUM)

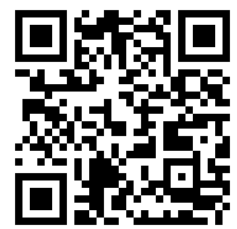

How to cite this article:

Davis LC, Baumer TG, Bey MJ, Holsbeeck MV. Clinical utilization of shear wave elastography in the musculoskeletal system. Ultrasonography. 2019 Jan;38(1):2-12. 
strain ratio of tissue within a region of interest (ROI) in relation to surrounding tissues. It is currently utilized in the clinical evaluation of soft tissues that are relatively homogeneous in echotexture such as the liver, breast, and thyroid. Strain elastography has not been widely adopted for routine clinical use in the musculoskeletal system in the United States, at least insofar as we were able to gauge from a recent electronic survey of musculoskeletal radiology practices (Table 1).

Shear wave elastography (SWE) produces an acoustic radiofrequency force impulse, sometimes called an "acoustic wind," which generates transversely-oriented shear waves that propagate through surrounding tissue (Fig. 1). The velocity of the propagating shear waves is measured (m/sec) and a qualitative color map is produced. Theoretically, the shear modulus, or tissue elasticity, can then be calculated from the shear wave speed (SWS), providing important biomechanical information about tissue quality [4]. This objective data, acquired in a novel, non-invasive ultrasound examination, provides important clinical and prognostic information and has the potential to revolutionize bone and joint imaging.

Despite the excitement around SWE, its clinical application has been hindered by technical and artifactual challenges, and the technology currently remains primarily in "research mode," with

Table 1. Responses to survey questions regarding the availability and utilization of ultrasound elastography in the musculoskeletal system

\begin{tabular}{|c|c|}
\hline Question & No. (\%) \\
\hline \multicolumn{2}{|c|}{$\begin{array}{l}\text { Do you perform musculoskeletal ultrasound at your } \\
\text { institution? }\end{array}$} \\
\hline Yes & $146(89.5)$ \\
\hline No & $17(10.4)$ \\
\hline \multicolumn{2}{|c|}{$\begin{array}{l}\text { Is ultrasound elastography available at your } \\
\text { institution (in any body system)? }\end{array}$} \\
\hline Yes & $85(52.2)$ \\
\hline No & 65 (39.9) \\
\hline Unsure & $13(8.0)$ \\
\hline \multicolumn{2}{|c|}{$\begin{array}{l}\text { If elastography is available, which of the following } \\
\text { options are available? }\end{array}$} \\
\hline Pressure/strain & $45(52.9)$ \\
\hline Shear wave & $40(47.1)$ \\
\hline \multicolumn{2}{|c|}{$\begin{array}{l}\text { If elastography is available, do you utilize it in the } \\
\text { musculoskeletal system for clinical purposes? }\end{array}$} \\
\hline Yes & $10(11.8)$ \\
\hline No & 75 (88.2) \\
\hline \multicolumn{2}{|c|}{$\begin{array}{l}\text { If elastography is available, do you utilize it in the } \\
\text { musculoskeletal system for research purposes? }\end{array}$} \\
\hline Yes & $22(25.9)$ \\
\hline No & $63(74.1)$ \\
\hline
\end{tabular}

slow progress in the clinical realm over the past 15 years. The aims of this paper are to review the current state of shear wave imaging in the musculoskeletal system, to discuss the challenges related to image acquisition, to review technical obstacles, and to propose a path forward for SWE in the musculoskeletal system.

\section{Current State of Shear Wave Elastography}

Despite progress in the laboratory setting, the utilization of SWE in clinical practice is still in its infancy. An electronic survey, created via SurveyMonkey (SurveyMonkey Inc., San Mateo, CA, USA; http:// www.surveymonkey.com) was sent to musculoskeletal radiologists throughout the United States who were members of the Society of Skeletal Radiology. Of the 163 respondents to the survey (Table 1), $85(52.15 \%)$ stated that ultrasound elastography (either strain or shear wave) was available at their institution. Of those, 45

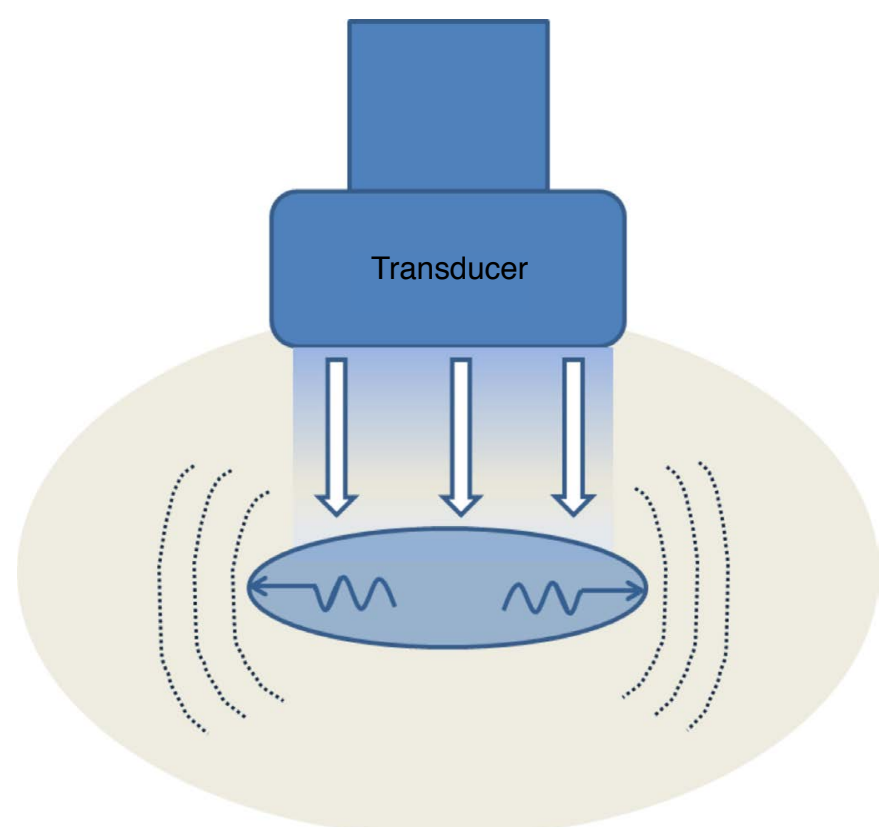

Fig. 1. Simplified illustration of shear wave elastography demonstrating the application of an acoustic radiofrequency impulse (ARFI, arrows) and the horizontal propagation of shear waves in the tissue (horizonal waves and interrupted curved lines). After the ARFl is applied, the transducer measures the velocity of the shear waves as they propagate through tissue, perpendicular to the original ARFI. From this information, a qualitative color map is created and a quantitative shear wave speed (SWS) is measured $(\mathrm{m} / \mathrm{sec})$. From the SWS, tissue elasticity can be calculated using the Young modulus, but it is important to consider that this calculation assumes an isotropic tissue with uniform density, and is therefore inaccurate in assessing in vivo tissues. For this reason, quantitative shear wave measurements in the musculoskeletal system are often reported as velocity or SWS, rather than tissue elasticity ( $\mathrm{KPa})$. 
respondents (52.94\%) acknowledged the specific availability of SWE, a greater number than had strain elastography available $(n=40$, 47.10\%).

Despite the fact that elastography technology was available for approximately half of the respondents, it was reported not to be widely used. In our survey, 22 respondents (25.88\%) acknowledged utilization of any form of elastography for research purposes, while only $10(11.76 \%)$ reported utilizing any form of elastography for clinical purposes. When queried about specific utilization of SWE, only five respondents $(5.88 \%)$ stated that they utilized SWE in a clinical setting, and all five of those respondents worked in academic settings.

The reasons for the delayed clinical implementation of SWE in the musculoskeletal system are multifactorial but relate, at least in part, to difficulty obtaining reliable SWS measurements. While previous studies have demonstrated excellent inter-reader repeatability in the skin [5], and almost perfect inter-reader repeatability in the breast [6] and liver [7], results in the musculoskeletal system have been much more varied. Intra-user repeatability has been reported as good to very good (intra-class correlation coefficient [ICC] $>0.6$ ) in the majority of studies on both tendons and muscles. There are, however, still many discrepancies in inter-user repeatability (Table 2). While inter-user repeatability was reported to be good to very good (ICC >0.6) for the gastrocnemius [8-10], tibialis anterior [11], and supraspinatus [12,13] muscles and for the flexor pollicis longus [14], quadriceps [14], patellar [15], and intramuscular supraspinatus [12] tendons, it was only reported as fair to moderate $(0.2<\mid \mathrm{ICC}<0.6)$ for the abdominal wall muscles [16] and the Achilles [14] and patellar tendons [14].

Despite a fairly substantial body of work supporting inter-user and intra-user repeatability across a wide range of musculoskeletal tissues, the reliability of day-to-day measurements is less well studied and remains poorly understood (Table 2). Some studies have shown that, even with good or very good intra-reader and interreader repeatability, inter-day repeatability may be poor [12]. Only a few studies have shown good to very good inter-day repeatability

Table 2. Reported intra-user, inter-user, and inter-day repeatability for shear wave measurements organized by author, the tissue being analyzed, and repeatability values

\begin{tabular}{|c|c|c|c|c|}
\hline Tissue & Intra-user repeatability & Inter-user repeatability & Inter-day repeatability & Study \\
\hline Biceps brachii & $0.89-0.94$ & - & - & Nordez et al. (2008) [17] \\
\hline Gastrocnemius muscle & $0.65-0.99$ & - & - & Maisetti et al. (2012) [9] \\
\hline Medial gastrocnemius muscle & - & 0.99 & - & Chernak et al. (2013) [8] \\
\hline Achilles tendon & - & $0.00^{a)}-0.82$ & - & Peltz et al. (2013) [14] \\
\hline Patellar tendon & - & $0.23^{\mathrm{a})}-0.90$ & - & \\
\hline Quadriceps tendon & - & $0.61-0.87$ & - & \\
\hline Flexor pollicis longus tendon & - & $0.43^{\mathrm{b})}-0.92$ & - & \\
\hline Biceps brachii muscle & $0.92-0.99$ & - & $0.98-0.99$ & Yoshitake et al. (2014) [18] \\
\hline Tibialis anterior muscle & $0.85-0.97$ & - & - & Koo et al. (2014) [11] \\
\hline Patellar tendon & $0.83-0.99$ & $0.74-0.82$ & - & Hsiao et al. (2015) [15] \\
\hline Posterior capsule, shoulder & 0.94 & 0.79 & - & Takenaga et al. (2015) [19] \\
\hline Posterior-inferior capsule, shoulder & 0.93 & 0.76 & - & \\
\hline Supraspinatus muscle & $0.70-0.80$ & 0.89 & - & Rosskopf et al. (2016) [13] \\
\hline Medial head gastrocnemius muscle & $0.94-0.99$ & $0.84-0.96$ & $0.83-0.90$ & Nakamura et al. (2016) [10] \\
\hline Achilles tendon & $0.80-0.85$ & $0.585^{b)}$ & - & Siu et al. (2016) [20] \\
\hline Patellar tendon & $0.91-0.92$ & 0.71 & $0.81-0.83$ & Tas et al. (2017) [21] \\
\hline Rectus femoris muscle & $0.93-0.94$ & 0.95 & $0.81-0.91$ & \\
\hline Supraspinatus muscle (passive) & 0.91 & 0.73 & $0.33^{\mathrm{a})}$ & Baumer et al. (2017) [12] \\
\hline Supraspinatus tendon (passive) & 0.87 & 0.74 & $0.48^{\mathrm{d})}$ & \\
\hline Supraspinatus muscle (active) & 0.90 & 0.79 & 0.65 & \\
\hline Supraspinatus tendon (active) & 0.94 & 0.97 & 0.94 & \\
\hline
\end{tabular}

Note the wide variation in repeatability, particularly inter-user repeatability, in multiple different tissues.

a) Poor values of the intra-class correlation coefficient $(I C C)(<0.4)$. ${ }^{\text {b) }}$ Fair values of the ICC $(0.4-0.59)$. ${ }^{\text {c }}$ Siu et al. [20] reported the ICC $(1, k)$ and ICC $(2, k)$ values, while all other studies reported ICC $(1,1)$ and ICC $(2,1)$ values. ${ }^{d)}$ Moderate values of the ICC $(0.60-0.74)$. 
(ICC $>0.6)$ for the medial gastrocnemius [10], rectus femoris [21], and supraspinatus [12] muscles and the patellar [21] and intramuscular supraspinatus [12] tendons.

As an emerging technology, there is significant variation in the study design, study implementation, and, not surprisingly, published results of SWE research. Additionally, SWE measurements made using standardized ultrasound phantoms with standardized methods demonstrated statistically significant differences in SWS estimations among systems and depending on the depth of the object [3]. Given these inconsistencies, it is not surprising that SWE has not made any permanent inroads into mainstream musculoskeletal imaging.

\section{Challenges Inherent to Musculoskeletal Tissue}

\section{Musculoskeletal Tissue Heterogeneity}

The background tissues in body systems where SWE is widely used and accepted, such as the liver, skin, and breast, are relatively homogeneous when compared to tissues in the musculoskeletal system. One possible explanation for discrepancies in SWE measurements in the musculoskeletal system is the inherent heterogeneity of musculoskeletal tissues. Individual skeletal muscle fibers are surrounded by endomysium, organized into fascicles that are surrounded by perimysium, and grouped into a muscle belly that is surrounded by epimysium. Nerve, arteries, veins, and lymphatic vessels course between muscle fascicles throughout the muscle. This intramuscular vasculature has been shown to affect SWE measurements, with higher measured SWS values around vessels than in other areas of the medial head gastrocnemius muscle; the vasculature also alters color maps, with more variation in color maps when vessels are included in the field of view [22]. These findings are exacerbated on short-axis imaging, as vessels are more difficult to visualize and may be inadvertently included in the field of view.

Traditionally in musculoskeletal imaging, long-axis and shortaxis images are obtained in relation to the structures being imaged. Depending on the specific muscle of interest, the individual muscle fibers may be parallel or obliquely oriented in relation to the long axis of the muscle. In pennate muscles, an aponeurosis runs along the superficial margins of the muscle, attaching the muscle to the tendon. If all muscle fascicles are on the same side of the tendon, the muscle is classified as a unipennate muscle. If, however, there is a single central tendon with muscle fascicles on both sides of the tendon tissue, the muscle is classified as bipennate, and if there are multiple central tendons with multidirectional muscle fibers, it is classified as multipennate. Given these variations in muscle architecture, the orientation of the transducer while positioned for long-axis imaging of the muscle belly may result in "off-axis" or oblique imaging of individual muscle fibers, a factor that may affect shear wave measurements.

\section{Background Tissue Activity}

Although not widely studied, the underlying state of the muscle or tendon may affect SWS measurements. While the stiffness of muscles increases during contraction, probably due to increased cross-bridge formation [18], underlying edema or inflammation may also affect SWS measurements. Most SWE studies have been performed on passive muscles and tendons, but some studies have focused on changes in SWS measurements with muscle activation and have demonstrated a linear relationship between increasing SWS measurements and progressive isometric contraction of the biceps brachii, suggesting that SWE is a feasible modality for quantifying muscle stiffness $[17,18,23]$.

While differences in SWS measurements have been observed between active and passive muscles, the exact relationship remains unclear $[8,24]$. Interestingly, a study by Baumer et al. [24] measured SWS in the supraspinatus tendon and muscle at rest (passive) and with minimal muscle activation (slight abduction of the arm). They found that the active SWS measurements were greater than the passive SWS measurements in both the muscle and tendon, in agreement with previously published studies. In addition, while both passive and active repeatability measurements on a single day were very good, there was only fair repeatability in passive tissue from day to day. In contrast, active tissue displayed much greater dayto-day repeatability. While the reasons for the variations in passive tissue SWS measurements are not fully understood, it is possible that passive tissue may be more sensitive to changes in mechanical loading associated with activities of daily living; standardizing this from day to day may not be feasible and may account for the dayto-day variations in measurements. It is possible that mild activation of the muscle and tendon helps standardize the tissue state, resulting in improved day-to-day repeatability, which could possibly increase the accuracy of longitudinal studies.

\section{Tissue Depth and Overlying Soft Tissues}

Several studies have investigated the effect of tissue depth on SWS measurements. An in vivo study [25] demonstrated that the average SWS in the vastus lateralis muscle did not change with imaging depth within the muscle, although the variance within the ROI increased as depth increased. Another study by Dillman et al. [26] revealed that phantoms with SWS measured between 0.5 and $3 \mathrm{~m} / \mathrm{sec}$ showed little change in SWS measurements with changes in depth.

To illustrate and expand upon these findings, we created a "soft" (5\% gelatin) and a "stiff" (10\% gelatin) phantom using water, unflavored gelatin, and psyllium hydrophilic mucilloid fiber, as detailed by Bude and Adler [27]. The phantoms were then imaged, 
with the deepest aspect of the ROI box positioned from 3 to $6 \mathrm{~cm}$, at $1 \mathrm{~cm}$ increments (Fig. 2A-D). The average SWS measured in the "soft" phantoms was $1.90 \mathrm{~m} / \mathrm{sec}$, a SWS that has been observed in passive muscle $[10,12,13]$. The average SWS measured in the "stiff" phantoms was $3.97 \mathrm{~m} / \mathrm{sec}$, a SWS that has been observed in active muscle or smaller tendons [12].

The soft phantom showed little change in SWS with increasing depth, although some variability in color was seen in the deeper ROI images. To confirm that this slight change in variability was related to depth of ROI and not settling of components of the phantom during preparation, the phantom was flipped $180^{\circ}$ (Fig. 2E) and reimaged. The results when the $\mathrm{ROI}$ was positioned over the same physical area, but closer to the transducer, demonstrated that the measurement errors in the phantom were indeed a function of depth and not a feature of phantom inhomogeneity.

The stiff phantom showed similar color maps with superficial

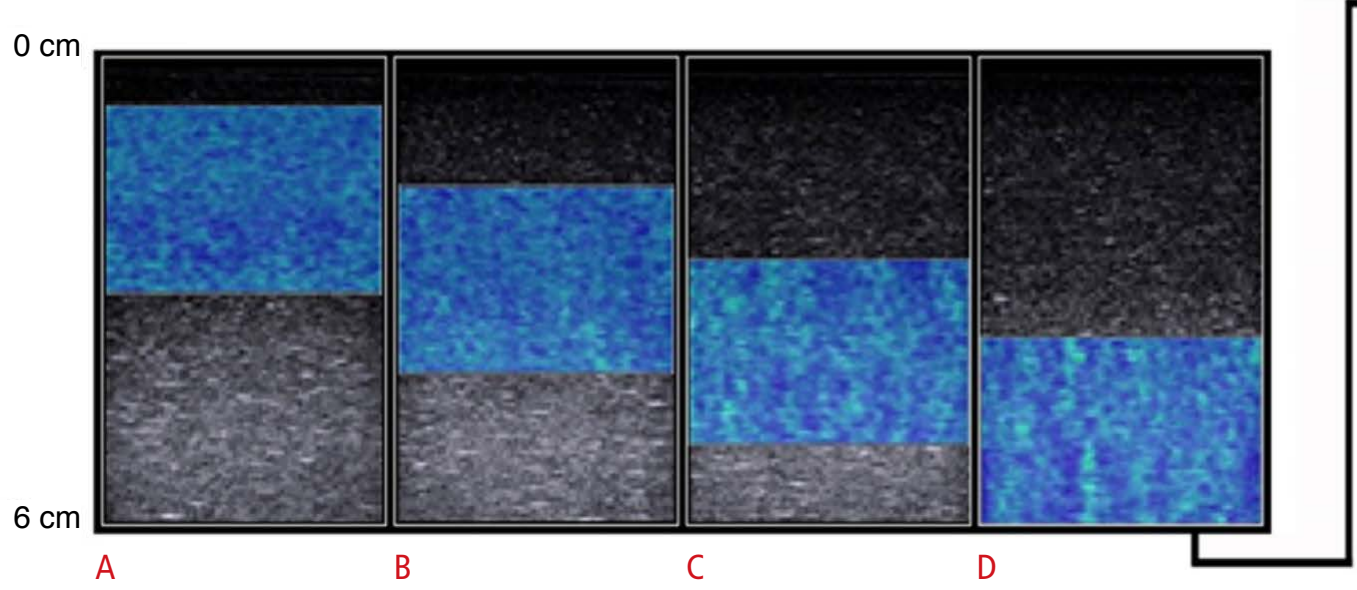

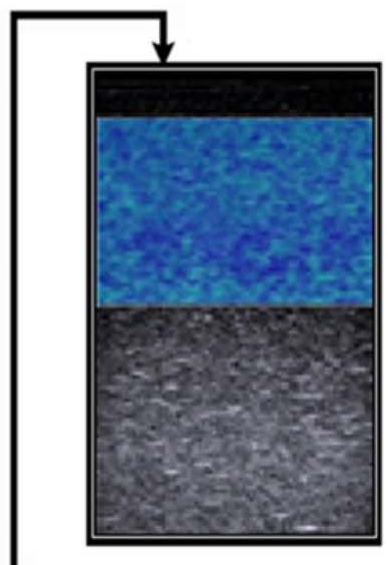

E

Fig. 2. Multiple shear wave elastography color maps of a soft phantom, whose shear wave speed (SWS) was consistent with passive muscle (average SWS $1.90 \mathrm{~m} / \mathrm{sec}, 5 \%$ gelatin), with the region of interest (ROI) box centered at depths of $2 \mathrm{~cm}(\mathrm{~A}), 3 \mathrm{~cm}$ (B), $4 \mathrm{~cm}(\mathrm{C})$, and $5 \mathrm{~cm}(\mathrm{D})$.

There is relatively uniform color in the superficial ROI (A, B), with a slight, progressive increase in color variability in the deeper ROI (C, D). To confirm that the variability was related to depth and not inherent to the phantom, the phantom was flipped $180^{\circ}$ and an additional color map was obtained (E), which demonstrated uniform color.

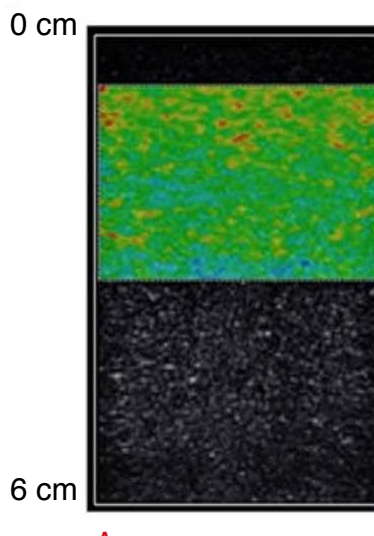

A

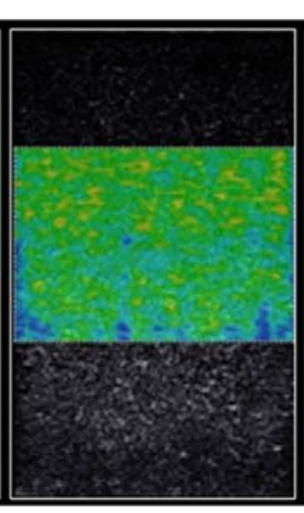

B

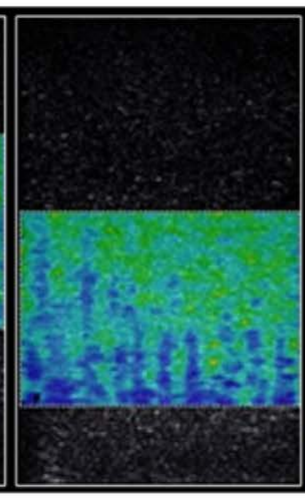

C

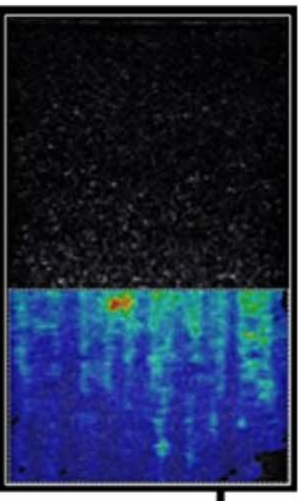

D

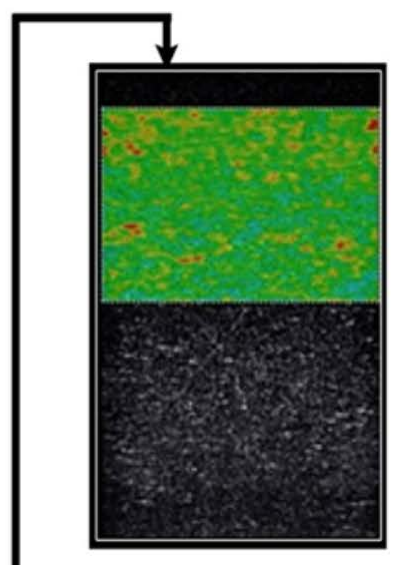

$\mathrm{E}$

Fig. 3. Multiple shear wave elastography color maps of a hard phantom, whose shear wave speed (SWS) was consistent with active muscle, (average SWS $3.97 \mathrm{~m} / \mathrm{sec}, 10 \%$ gelatin), with the region of interest (ROI) box centered at depths of $2 \mathrm{~cm}(\mathrm{~A}), 3 \mathrm{~cm}(\mathrm{~B}), 4 \mathrm{~cm}(\mathrm{C})$, and $5 \mathrm{~cm}(\mathrm{D})$.

The images demonstrate color variability at the deepest margins of the ROI, starting at a depth of approximately $4 \mathrm{~cm}(\mathrm{~B})$, which progressively worsened with deeper positioning of the ROI (C, D). To confirm that the variability was related to depth and not inherent to the phantom, the phantom was flipped $180^{\circ}$ and an additional color map was obtained (E), which demonstrated uniform color. 
positioning of the ROI (Fig. 2A, B), but variation in color was apparent when the ROI was positioned at $5 \mathrm{~cm}$ (Fig. 3C) and the SWS field changed almost completely at $6 \mathrm{~cm}$ (Fig. 3D). Again, to confirm that these changes were related to depth, and not settling of components of the phantom during preparation, the phantom was flipped $180^{\circ}$ (Fig. $3 \mathrm{E}$ ) and reimaged. The ROI positioned over the same physical area, but closer to the transducer, demonstrated that the measurements in the phantom were indeed a function of depth and not a feature of phantom inhomogeneity.

From these studies we can conclude that, in a phantom whose SWS was consistent with passive muscle, there was little to no effect of increasing depth on SWS measurements. A cadaveric study by Hatta et al. [28], in which the SWS of the supraspinatus was measured as overlying tissues were sequentially removed, also revealed no influence of overlying tissues on SWS measurements, further supporting the lack of influence on passive tissues. In a phantom whose SWS is consistent with active muscles or mediumsized tendons, however, artifactual changes in the SWS field begin at depths of $\sim 4 \mathrm{~cm}$; thus, although the depth of a passive muscle may not affect SWS, the influence of stiffer superficial layers is not yet fully understood.

\section{Underlying Osseous Structures}

In addition to the heterogeneity of musculoskeletal tissues, the tissues being imaged often directly overlie osseous structures, introducing an additional challenge to the acquisition of SWS measurements. While this seems to be a persistent obstacle in the musculoskeletal system and cannot be completely eliminated, the interference of underlying bones can be decreased by modifying the position of the patient and transducer.

Reflection artifacts are most easily identified when tissues are in a passive state. Long-axis B-mode imaging of the insertional supraspinatus tendon, as seen in Fig. $4 \mathrm{~A}$, and the corresponding color map, as seen in Fig. 4B, reveal inhomogeneity in SWS measurements within the supraspinatus tendon and overlying deltoid muscle, with low SWS (blue) and high SWS (red) measurements present in the same muscle and tendon. While it is possible that inhomogeneity such as this could be real, the linear alignment of these changes suggests that it represents a reflection artifact. This observation is based upon the fact that the image was obtained with the subject in the Crass position (shoulder internally rotated and slightly extended, elbow flexed $90^{\circ}$, forearm pronated and positioned behind the back). In this position, the deltoid is in a resting state and, barring substantial muscular pathology, is expected to exhibit relatively uniform SWS. Furthermore, the red and green colored areas are seen in the areas directly above prominent convex bony features, an area that we have termed the "reflective corridor" (Fig. 4C, corridor outlined in interrupted red lines). In this case, the displacement field generated by the SWE is substantially disrupted, such that even in areas between the main reflective

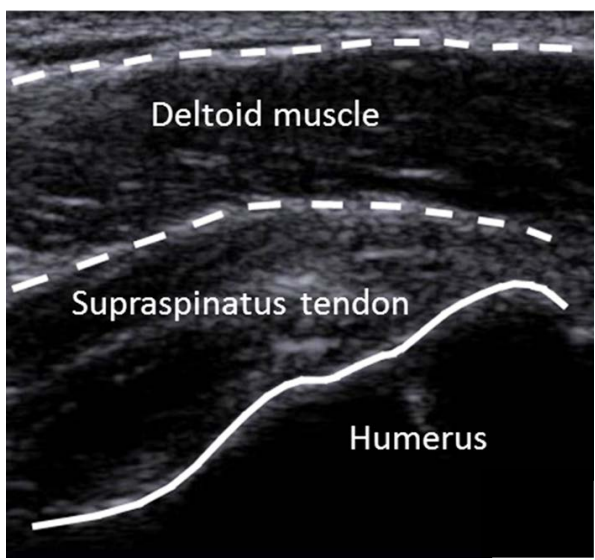

A

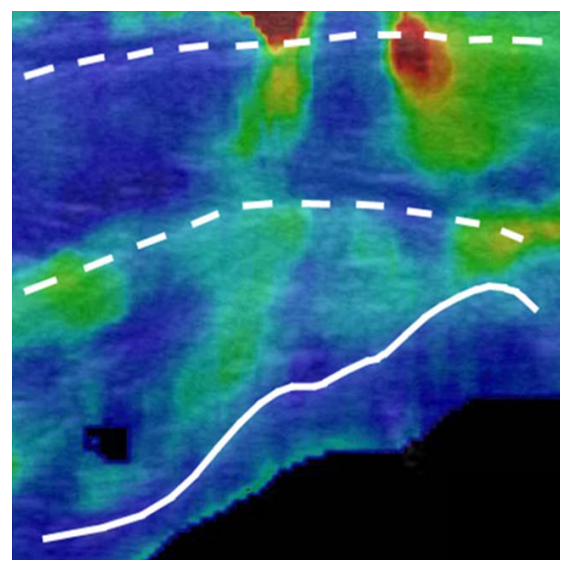

B

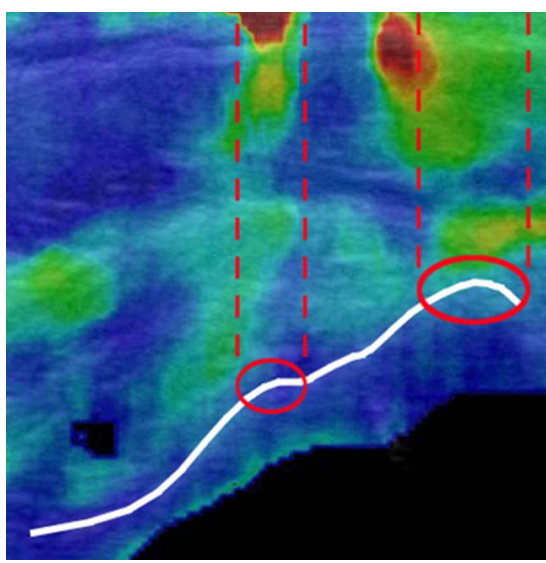

C

Fig. 4. Long-axis B-mode imaging of the insertional supraspinatus tendon and the corresponding color maps of shear wave elastography.

Gray-scale sonographic image of the insertional supraspinatus tendon (A), demonstrating the overlying deltoid muscle and the bony acoustic landmark of the underlying greater tuberosity with the patient in the typical Crass position (far internal shoulder rotation, flexed elbow, pronated forearm) and the transducer oriented along the long axis of the tendon. Color maps produced during shear wave elastography imaging of the same region $(B, C)$ demonstrate some vertical, linear bands of signal abnormality in the supraspinatus tendon and deltoid muscle, extending to the superficial soft tissues which are contained within dashed parallel lines in C. While these changes could represent inhomogeneity of tendon/muscle, the linear pattern of signal change and the focal osseous convex margins deep to these areas, depicted by red circles in C, suggest that the signal changes were artifactual, representing a "reflective corridor." 
corridors, the accuracy of the measurements may be unreliable.

Fortunately, this reflection artifact can be minimized. In our experience, convex surfaces generate the most substantial interruptions to the displacement field, while concave surfaces tend to generate more localized disruptions, although this has not yet been fully evaluated. While it may be impossible to eliminate reflection entirely, depending on the tissue being observed, it may be possible to shift the reflection corridor to a less important area within the field of view. For example, in Fig. 5, the transducer orientation was changed slightly to view the supraspinatus tendon insertion site in a non-traditional, slightly oblique orientation. With this slight change in the transducer position, the underlying bony backdrop was minimized, as the convex superficial bony margins were shifted to the periphery of the field of view. The images in Fig. 5 are from the same subject as above and nicely illustrate how the corresponding SWE image is much more homogeneous in both the supraspinatus tendon and overlying deltoid muscle. Often, as in this case, the convex bony features are not entirely avoidable, but can be shifted to the lateral margins of the ROI, creating usable, reliable data within the area between the reflective corridors.

Admittedly, even these optimized images demonstrate inhomogeneity, as shown by the presence of some subtle streaks inside the tendon that may be artifactual and related to reflection; however, smaller reflections like these may be eliminated as machine settings and imaging algorithms continue to advance.

\section{Potential Pitfalls and Technical Considerations}

\section{Transducer Positioning}

The position of the ultrasound transducer is a modifiable variable that should be considered when performing SWE. Shear waves propagate more readily along muscle fibers when the transducer is oriented longitudinally, rather than perpendicular or at a $45^{\circ}$ oblique angle $[29,30]$ to the tendon, and SWE measurements obtained parallel to the muscle fibers increase, as expected, with increasing tensile load [29].

Chino et al. [22] obtained transverse and longitudinal shear wave measurements of the biceps brachii, a unipennate muscle, and the medial head gastrocnemius, a bipennate muscle. The results from these studies demonstrated more similar measurements in the transverse and longitudinal planes when scanning the medial head gastrocnemius and more variable measurements when scanning the biceps brachii. The differences in measurements were likely related to the underlying muscle fiber orientation, as shear waves propagate more quickly along the parallel (unipennate) muscle fibers of the biceps brachii, resulting in high SWS when imaged in the longitudinal plane and a significantly lower SWS when imaged in the transverse plane. Conversely, the muscle fibers of the medial head gastrocnemius are oriented oblique to the central tendon, and are therefore obliquely oriented in both the traditional longitudinal and transverse imaging planes. Consequently, the medial head

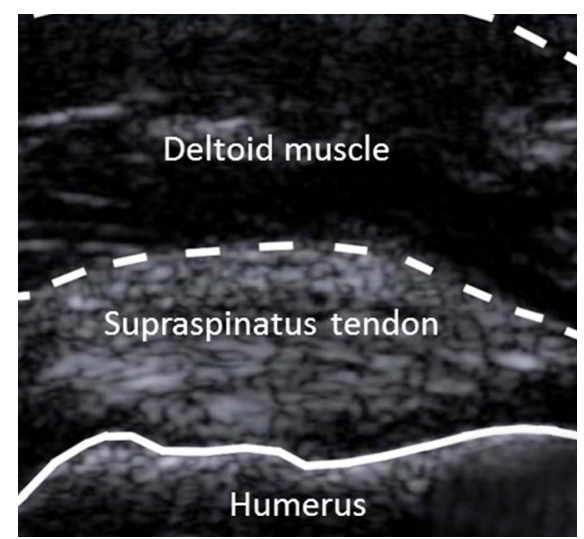

A

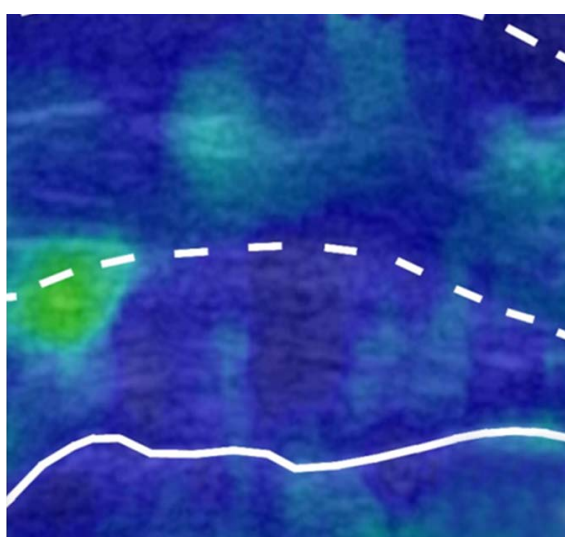

B

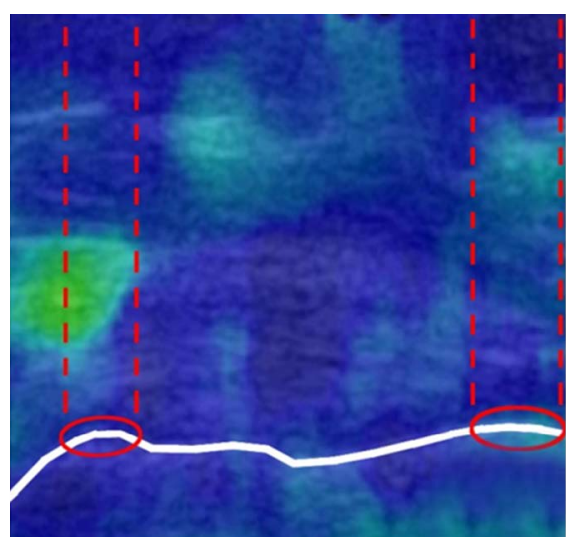

C

Fig. 5. Oblique-axis B-mode imaging of the insertional supraspinatus tendon and the corresponding color maps of shear wave elastography.

Gray-scale sonographic image of the insertional supraspinatus tendon (A), the overlying deltoid muscle, and the bony acoustic landmark of the underlying greater tuberosity with the patient in the typical Crass position (far internal shoulder rotation, flexed elbow, pronated forearm), but with the transducer orientation slightly oblique to the long axis of tendon. Color maps produced during shear wave elastography imaging of the same region $(B, C)$ again demonstrate some vertical, linear areas of inhomogeneity, contained within dashed parallel lines in C, located superficial to focal osseous convex margins, depicted by red circles in C. When compared to the images in Fig. 4, the signal changes in these images are much less pronounced and the "reflective corridors" are shifted to the lateral margins of the field of view, minimizing their effect on shear wave speed measurements. 
gastrocnemius SWS measurements were more similar in the longitudinal and transverse planes.

The importance of longitudinal probe positioning has since been confirmed for the medial gastrocnemius, rectus femoris, biceps brachii, and rectus abdominus muscles, where images obtained with the transducer positioned transverse (or perpendicular) to the long axis of the muscle demonstrated lower image stability (i.e., increased color distribution throughout the image) than those obtained with the transducer positioned longitudinal (or parallel) to the long axis of the muscle [22].

\section{Transducer Pressure}

During traditional ultrasound scanning, the transducer applies firm, consistent pressure to the skin surface. When working with SWE,

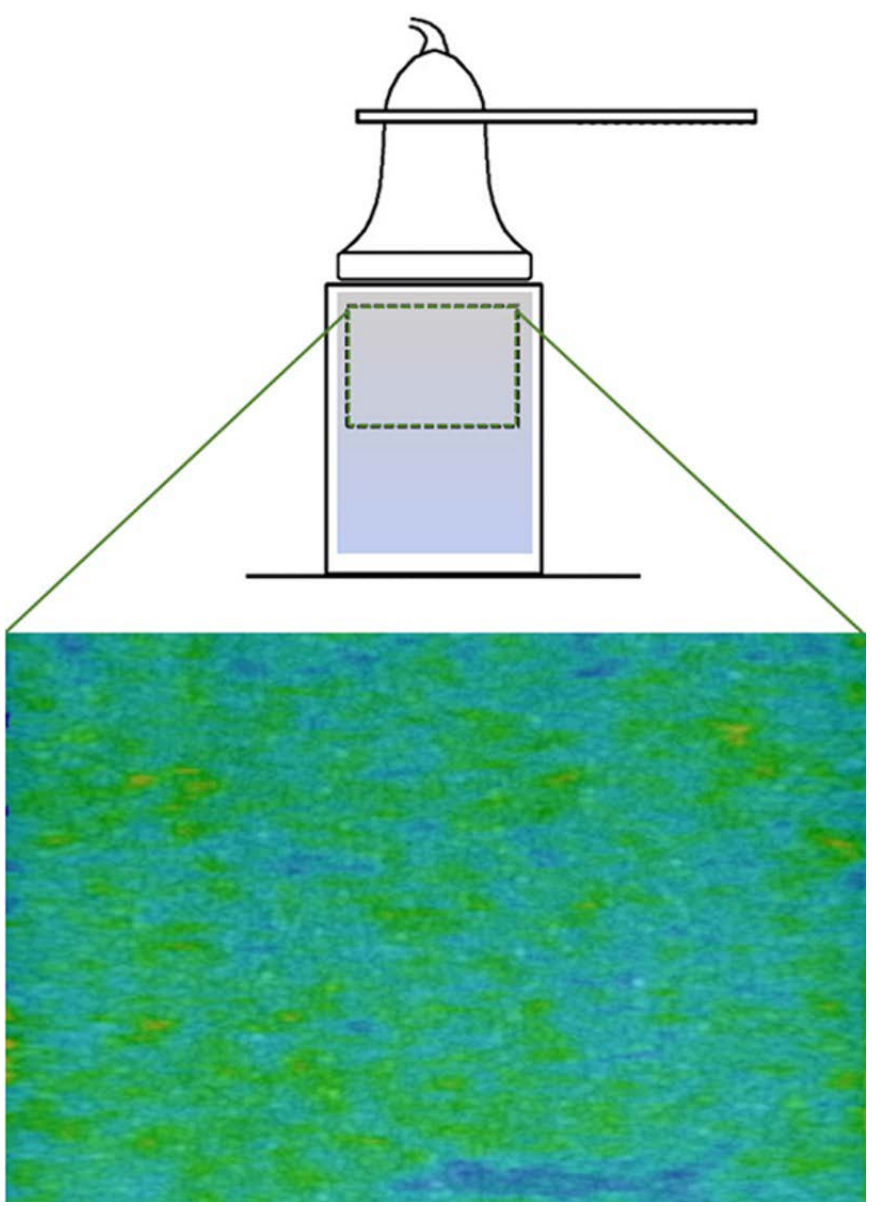

Fig. 6. Diagram illustrating an ultrasound probe that was fixed in place, a gel phantom that was also fixed in place (outlined by a rectangle) and the resultant shear wave elastography color map. In this case, the shear wave elastography color map is fairly uniform, with an average variance of $0.42 \mathrm{~m} / \mathrm{sec}$ and a measured shear wave speed of $3.97 \mathrm{~m} / \mathrm{sec}$. however, transducer pressure should be avoided or standardized as applied pressure compresses tissues below the surface. Tissue compression increases SWS measurements in all tissues, but the effect is particularly important in soft tissues where an increase in compression of $\sim 10 \%$ doubles SWS measurements [31]. In order to standardize measurements and avoid this induced variability in SWS measurement, consistent, light transducer pressure or a mounted transducer should be used during SWE imaging.

\section{Patient Positioning}

A good understanding of the functional musculoskeletal unit being imaged is important in SWE of the musculoskeletal system, specifically related to the strain behavior of soft tissues relative to joint position. Several studies have correlated increased

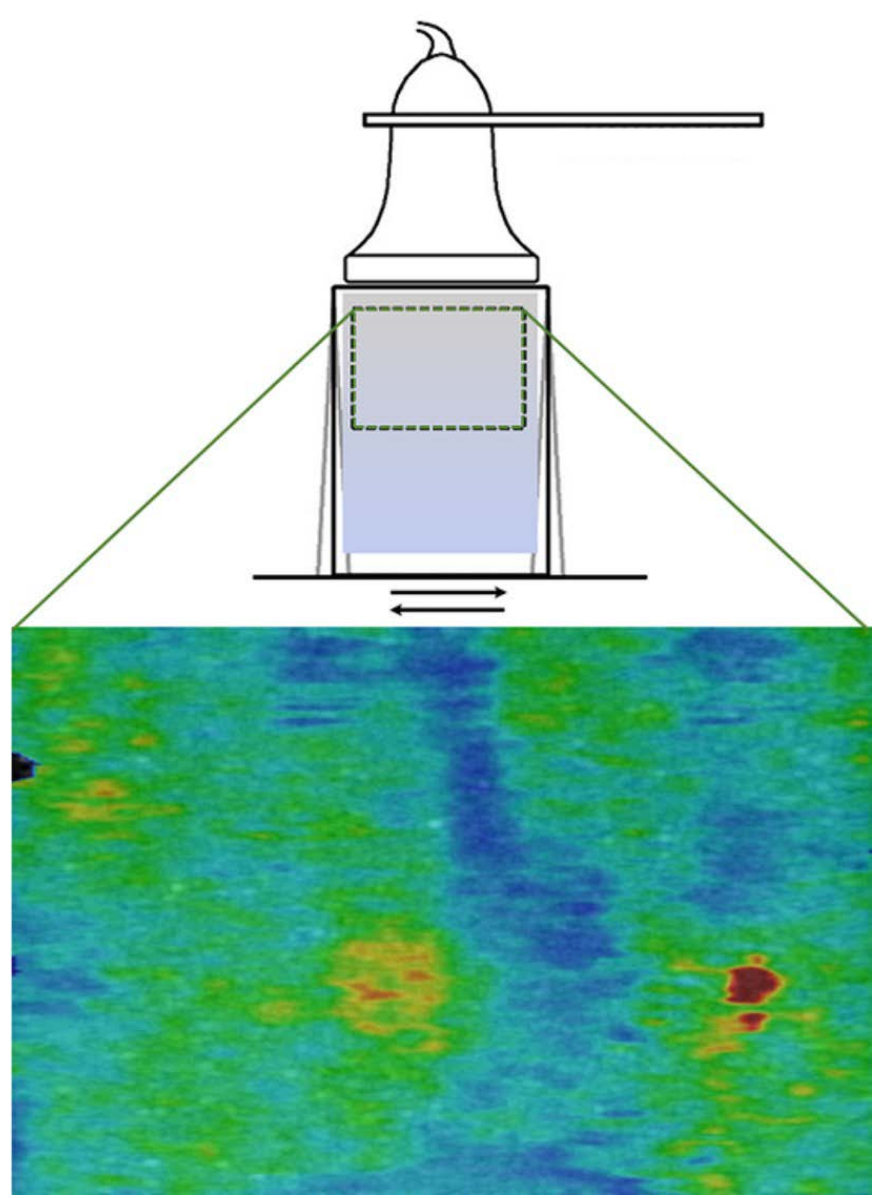

Fig. 7. Diagram illustrating an ultrasound probe that was fixed in place, with the same gel phantom as in Fig. 6, with induced motion indicated by horizontal arrows, and the resultant shear wave elastography color map of the same phantom. In this case, the shear wave elastography color map demonstrates nonuniformity, with the variance more than doubling to $0.86 \mathrm{~m} / \mathrm{sec}$ and the shear wave speed dropping to $3.32 \mathrm{~m} / \mathrm{sec}$. 
tension in the tissue with increased SWS measurements [23,3235]. In a cadaveric study, a large increase in strain over a small range of flexion was observed in the patellar tendon. Specifically, when flexion minimally increased from $20^{\circ}$ to $30^{\circ}$, strain disproportionately increased from $\sim 2 \%$ to $\sim 6 \%$. The same study also documented fairly static strain in the patellar tendon from $30^{\circ}$ to $50^{\circ}$ flexion, which is an important detail to remember when studying this tendon [36].

As SWS measurements vary with changes in tissue state, and alterations in joint position result in changes in tissue state, it is likely that SWS changes are affected by joint position, although this information has not been integrated into clinical imaging, to our knowledge. A more rigorous study of the interactions among tissue strain, joint position, and SWS measurements is necessary to draw a firm conclusion, but the current evidence suggests that joint positions that display rapid strain changes are not ideal positions for SWE imaging.

\section{Decreasing Motion}

One of the primary artefacts that may appear in SWE relates to movement. This motion artifact can either be due to movement of the probe relative to the subject (user-induced) or movement of the tissue relative to the probe (subject-induced). Motions that can influence SWS readings can be as subtle as a muscle twitch during the acquisition sequence. Regardless of the origin, motion has an impact on the quantitative results obtained during SWE imaging.

To demonstrate the effect of a small movement on the SWS field calculations, a simple study using a "soft" gelatin phantom ( $5 \%$ gelatin) was performed. In the first condition, both the phantom and the ultrasound probe were fixed in place and several SWE images were acquired (Fig. 6). In these trials, the phantom appeared to be fairly uniform, with an average SWS of $3.97 \mathrm{~m} / \mathrm{sec}$ and an average intra-image variance (standard deviation/mean) of $0.42 \mathrm{~m} / \mathrm{sec}$. In the second condition, the probe was held fixed in place, but a small amount of motion was induced in the gelatin by generating a smallmagnitude translation at the end furthest from the probe (Fig. 7). In this case, the SWS field demonstrated areas of high and low SWS where none had been observed before the induced motion. This motion altered SWS substantially, with the average SWS dropping to $3.32 \mathrm{~m} / \mathrm{sec}$ and the intra-image variance more than doubling to $0.86 \mathrm{~m} / \mathrm{sec}$.

The characteristics observed in this simple study have also been observed during in vivo trials from motions as simple as a deep breath or a palpable muscle spasm. It is important to realize the possibility of this artifact and to control for it as best as possible. Multiple images taken with the participant and user both aiming to remain still will help eliminate motion artifacts. Furthermore,

Table 3. Summary of challenges encountered in SWE imaging of the musculoskeletal system, with suggestions for avoiding or minimizing each challenge

\begin{tabular}{|c|c|}
\hline Challenge & Suggestion \\
\hline $\begin{array}{l}\text { Tissue depth and overlying soft } \\
\text { tissues }\end{array}$ & $\begin{array}{l}\text { In tissue (either phantom or cadaveric) whose SWS is consistent with passive muscle, there is little to no effect of } \\
\text { increasing depth on SWS measurements (Hatta). } \\
\text { The influence of stiffer superficial tissue layers remains unknown. }\end{array}$ \\
\hline Background tissue activity & $\begin{array}{l}\text { There is a linear relationship between increasing SWS measurements and progressive isometric contraction of the } \\
\text { biceps brachii, suggesting that SWE is a feasible modality for quantifying muscle stiffness (Yoshitake, Yavuz, Nordez). } \\
\text { Mild activation of the muscle may help standardize the tissue state, resulting in improved day-to-day repeatability. }\end{array}$ \\
\hline Underlying osseous structures & $\begin{array}{l}\text { Vertical, linear signal change overlying focal osseous convex margins likely represent "reflective corridors." } \\
\text { Consider shifting the orientation or the transducer slightly to move the convex osseous margins to the peripheral } \\
\text { margins of the field of view, which may minimize their impact on SWS measurements. }\end{array}$ \\
\hline Transducer positioning & $\begin{array}{l}\text { Long-axis imaging improves visualization of intramuscular vasculature, which should be excluded from SWS } \\
\text { measurements when possible (Chino). } \\
\text { Knowledge of the muscle architecture may allow for improved transducer positioning for long-axis imaging of muscle } \\
\text { fascicles, although this may not be possible in multipennate muscles. }\end{array}$ \\
\hline Transducer pressure & SWS measurements can be altered by transducer pressure; very light pressure may minimize these changes. \\
\hline Patient positioning & $\begin{array}{l}\text { Strain of a tendon affects SWS measurement and even small changes in joint position may result in large changes in } \\
\text { strain (Sheehan). } \\
\text { SWS changes related to strain appear to be less significant when the tissue is under no strain or very high strain. } \\
\text { Consider placing participants in a position where there is either no tension or clearly high tension. }\end{array}$ \\
\hline Decreasing motion & $\begin{array}{l}\text { Both subject-induced and operator-induced motion affects SWS measurements. } \\
\text { Consider obtaining multiple images with the participant and user both aiming to remain still. } \\
\text { Analysis of repeated images will help identify any trials where an involuntary motion did occur. }\end{array}$ \\
\hline
\end{tabular}

SWE, shear wave elastography; SWS, shear wave speed. 
repeated imaging will help identify any trials where an involuntary motion did occur, as shown either by an image appearing different from the other trials or by the SWS being different. As in other imaging modalities, it may be good practice to ask participants to inhale and hold their breath for the duration of each image acquisition.

\section{Data Analysis and Reporting Suggestions}

\section{"Shear Wave Speed" versus "Tissue Elasticity"}

The calculation of tissue elasticity is based upon the assumptions that the soft tissues are elastic, incompressible, homogeneous, and isotropic [3]. The SWS measured during SWE is only a portion of the calculation of the Young modulus, which is needed to determine tissue elasticity, as $E=3 \mu=3 p c T^{2}$, where $E=$ the Young modulus, $\mu=$ the shear modulus, $\mathrm{p}=$ tissue density, and $\mathrm{cT}=$ the speed of transversely propagated waves, or shear waves [37]. With this relationship, unless the tissue density is $1 \mathrm{~g} / \mathrm{mm}^{3}$, the SWS does not correspond exactly with the Young modulus [3]. The musculoskeletal system, with its viscoelastic, heterogeneous, anisotropic tissues, presents inherent challenges to calculating tissue elasticity using the Young modulus. As a result, SWE measurements in the musculoskeletal system should be presented in terms of SWS $(\mathrm{m} /$ $\mathrm{sec})$, rather than tissue elasticity $(\mathrm{kPa})$.

\section{Conclusion}

SWE, while promising in theory, requires further advances in technology and a better understanding of image acquisition before it can reliably be used for musculoskeletal imaging. The musculoskeletal system is composed of heterogeneous, anisotropic tissue, including muscles as well as complex muscle-tendonenthesis-bone interfaces that make the implementation of SWE challenging. Many of the stumbling blocks encountered during musculoskeletal SWE imaging are readily recognizable and can be overcome or minimized (Table 3); however, the effects of some obstacles on qualitative results are still not fully understood, despite ongoing research.

Continued collaboration between researchers and clinicians may enable the development of standardized imaging protocols and positions and may elucidate the causes of (and solutions for) imaging artifacts. Additionally, advancements in SWE transducer technology and more widespread distribution of SWE-capable machines may allow for a wider range of data acquisition.

ORCID: Leah C. Davis: https://orcid.org/0000-0003-4403-1098; Timothy G. Baumer: https://orcid.org/0000-0002-7722-0285; Michael J. Bey: https://orcid.org/0000-
0002-2638-5979; Marnix van Holsbeeck: https://orcid.org/0000-0001-5756-5617

\section{Conflict of Interest}

Siemens Healthcare provides Marnix van Holsbeeck with ultrasound equipment. All other authors have no competing interests.

\section{References}

1. Kane D, Grassi W, Sturrock R, Balint PV. A brief history of musculoskeletal ultrasound: from bats and ships to babies and hips. Rheumatology (Oxford) 2004;43:931-933.

2. Nazarian LN. The top 10 reasons musculoskeletal sonography is an important complementary or alternative technique to MRI. AJR Am J Roentgenol 2008;190:1621-1626.

3. Ryu J, Jeong WK. Current status of musculoskeletal application of shear wave elastography. Ultrasonography 2017;36:185-197.

4. Jeong WK, Lim HK, Lee HK, Jo JM, Kim Y. Principles and clinical application of ultrasound elastography for diffuse liver disease. Ultrasonography 2014;33:149-160.

5. Sun Y, Ma C, Liang X, Wang R, Fu Y, Wang $S$, et al. Reproducibility analysis on shear wave elastography (SWE)-based quantitative assessment for skin elasticity. Medicine (Baltimore) 2017;96:e6902.

6. Cosgrove DO, Berg WA, Dore CJ, Skyba DM, Henry JP, Gay J, et al. Shear wave elastography for breast masses is highly reproducible. Eur Radiol 2012:22:1023-1032.

7. Suh CH, Kim SY, Kim KW, Lim YS, Lee SJ, Lee MG, et al. Determination of normal hepatic elasticity by using real-time shearwave elastography. Radiology 2014;271:895-900.

8. Chernak LA, DeWall RJ, Lee KS, Thelen DG. Length and activation dependent variations in muscle shear wave speed. Physiol Meas 2013;34:713-721.

9. Maisetti O, Hug F, Bouillard K, Nordez A. Characterization of passive elastic properties of the human medial gastrocnemius muscle belly using supersonic shear imaging. J Biomech 2012;45:978-984.

10. Nakamura M, Ikezoe T, Umegaki H, Kobayashi T, Nishisita $S$, Ichihashi N. Shear elastic modulus is a reproducible index reflecting the passive mechanical properties of medial gastrocnemius muscle belly. Acta Radiol Open 2016;5:2058460115604009.

11. Koo TK, Guo JY, Cohen JH, Parker KJ. Quantifying the passive stretching response of human tibialis anterior muscle using shear wave elastography. Clin Biomech (Bristol, Avon) 2014;29:33-39.

12. Baumer TG, Davis L, Dischler J, Siegal DS, van Holsbeeck M, Moutzouros $\mathrm{V}$, et al. Shear wave elastography of the supraspinatus muscle and tendon: repeatability and preliminary findings. Biomech 2017;53:201-204.

13. Rosskopf AB, Ehrmann C, Buck FM, Gerber C, Fluck M, Pfirrmann CW. Quantitative shear-wave US elastography of the supraspinatus muscle: reliability of the method and relation to tendon integrity and muscle quality. Radiology 2016;278:465-474. 
14. Peltz CD, Haladik JA, Divine G, Siegal D, van Holsbeeck M, Bey MJ. ShearWave elastography: repeatability for measurement of tendon stiffness. Skeletal Radiol 2013;42:1151-1156.

15. Hsiao MY, Chen YC, Lin CY, Chen WS, Wang TG. Reduced patellar tendon elasticity with aging: in vivo assessment by shear wave elastography. Ultrasound Med Biol 2015;41:2899-2905.

16. MacDonald D, Wan A, McPhee M, Tucker K, Hug F. Reliability of abdominal muscle stiffness measured using elastography during trunk rehabilitation exercises. Ultrasound Med Biol 2016;42:10181025.

17. Nordez A, Gennisson JL, Casari P, Catheline S, Cornu C. Characterization of muscle belly elastic properties during passive stretching using transient elastography. J Biomech 2008;41:23052311.

18. Yoshitake Y, Takai Y, Kanehisa H, Shinohara M. Muscle shear modulus measured with ultrasound shear-wave elastography across a wide range of contraction intensity. Muscle Nerve 2014;50:103113.

19. Takenaga T, Sugimoto K, Goto H, Nozaki M, Fukuyoshi M, Tsuchiya $A$, et. al. Posterior shoulder capsules are thicker and stiffer in the throwing shoulders of healthy college baseball players: a quantitative assessment using shear wave ultrasound elastography. Am J Sports Med 2015;43:2935-2942.

20. Siu WL, Chan CH, Lam CH, Lee CM, Ying M. Sonographic evaluation of the effect of long-term exercise on Achilles tendon stiffness using shear wave elastography. J Sci Med Sport 2016;19:883-887.

21. Tas S, Onur MR, Yilmaz S, Soylu AR, Korkusuz F. Shear wave elastography is a reliable and repeatable method for measuring the elastic modulus of the rectus femoris muscle and patellar tendon. J Ultrasound Med 2017;36:565-570.

22. Chino K, Kawakami $Y$, Takahashi H. Tissue elasticity of in vivo skeletal muscles measured in the transverse and longitudinal planes using shear wave elastography. Clin Physiol Funct Imaging 2017:37:394-399.

23. Yavuz A, Bora A, Bulut MD, Batur A, Milanlioglu A, Goya C, et al. Acoustic radiation force impulse (ARFI) elastography quantification of muscle stiffness over a course of gradual isometric contractions: a preliminary study. Med Ultrason 2015;17:49-57.

24. Baumer TG, Dischler J, Davis L, Labyed Y, Siegal DS, van Holsbeeck $M$, et al. Effects of age and pathology on shear wave speed of the human rotator cuff. J Orthop Res 2018;36:282-288.

25. Alfuraih AM, O'Connor P, Hensor E, Tan AL, Emery P, Wakefield RJ. The effect of unit, depth, and probe load on the reliability of muscle shear wave elastography: Variables affecting reliability of SWE. J
Clin Ultrasound 2018;46:108-115.

26. Dillman JR, Chen S, Davenport MS, Zhao H, Urban MW, Song P, et al. Superficial ultrasound shear wave speed measurements in soft and hard elasticity phantoms: repeatability and reproducibility using two ultrasound systems. Pediatr Radiol 2015;45:376-385.

27. Bude RO, Adler RS. An easily made, low-cost, tissue-like ultrasound phantom material. J Clin Ultrasound 1995;23:271-273.

28. Hatta T, Giambini H, Uehara K, Okamoto S, Chen S, Sperling JW, et al. Quantitative assessment of rotator cuff muscle elasticity: reliability and feasibility of shear wave elastography. J Biomech 2015;48:3853-3858.

29. Eby SF, Song P, Chen S, Chen Q, Greenleaf JF, An KN. Validation of shear wave elastography in skeletal muscle. J Biomech 2013;46:2381-2387.

30. Gennisson JL, Deffieux T, Mace E, Montaldo G, Fink M, Tanter M. Viscoelastic and anisotropic mechanical properties of in vivo muscle tissue assessed by supersonic shear imaging. Ultrasound Med Biol 2010;36:789-801.

31. Barr RG, Zhang Z. Effects of precompression on elasticity imaging of the breast: development of a clinically useful semiquantitative method of precompression assessment. J Ultrasound Med 2012;31:895-902.

32. Ates F, Hug F, Bouillard K, Jubeau M, Frappart T, Couade M, et al. Muscle shear elastic modulus is linearly related to muscle torque over the entire range of isometric contraction intensity. J Electromyogr Kinesiol 2015;25:703-708.

33. Aubry S, Nueffer JP, Tanter M, Becce F, Vidal C, Michel F. Viscoelasticity in Achilles tendonopathy: quantitative assessment by using real-time shear-wave elastography. Radiology 2015;274:821829.

34. Dubois G, Kheireddine W, Vergari C, Bonneau D, Thoreux P, Rouch $P$, et al. Reliable protocol for shear wave elastography of lower limb muscles at rest and during passive stretching. Ultrasound Med Biol 2015;41:2284-2291.

35. Hatta T, Giambini H, Sukegawa K, Yamanaka Y, Sperling JW, Steinmann SP, et al. Quantified mechanical properties of the deltoid muscle using the shear wave elastography: potential implications for reverse shoulder arthroplasty. PLoS One 2016;11:e0155102.

36. Sheehan FT, Drace JE. Human patellar tendon strain: a noninvasive, in vivo study. Clin Orthop Relat Res 2000;370:201-207.

37. Parker KJ, Fu D, Graceswki SM, Yeung F, Levinson SF. Vibration sonoelastography and the detectability of lesions. Ultrasound Med Biol 1998;24:1437-1447. 